\title{
On the nature of intersensory facilitation of reaction time
}

\author{
STAN C. A. M. GIELEN \\ Rijksuniversiteit Utrecht, Utrecht, The Netherlands \\ RICHARD A. SCHMIDT \\ University of California, Los Angeles, California \\ and \\ PIETER J. M. VAN DEN HEUVEL \\ Rijksuniversiteit Utrecht, Utrecht, The Netherlands
}

\begin{abstract}
Two experiments examined the RT to visual stimuli presented alone and when either auditory (Experiment 1) or kinesthetic (Experiment 2) stimuli followed the visual event by 50 or $65 \mathrm{msec}$, respectively. As has been found before, the RT to combined stimulus events was 20 to $40 \mathrm{msec}$ shorter than to visual events alone. While such results have generally been interpreted to mean that two sensory modalities are interacting, Raab's (1962) hypothesis of statistical facilitationthat the subject responds to that stimulus modality whose processing is completed first-is also possible. Using Raab's model, but with relaxed assumptions, the present experiments show that RT to combined stimulus events is more rapid than can be accounted for by statistical facilitation. Therefore, some intersensory interaction was probably occurring. The nature of these possible interactions and the status of the statistical-facilitation hypothesis are discussed.
\end{abstract}

Since the pioneering work of Todd in 1912, it has been generally found that the reaction time (RT) to a visual imperative stimulus is from 20 to $80 \mathrm{msec}$ shorter if it is paired with a simultaneous (or delayed by as much as $100 \mathrm{msec}$ ) auditory stimulus (called an accessory stimulus), even though the subject is instructed not to respond to the accessory. When such effects are found, it is generally assumed that visual and auditory channels are interacting in some way to facilitate RT, and thus such effects tend to come under the label of intersensory facilitation. Todd's work has been repeated and extended a number of times, and today there is evidence that facilitation occurs in both simple- and choice-RT paradigms (Bernstein, Clark, Edelstein, 1969a; Bernstein \& Edelstein, 1971; Morrell, 1968b; Simon \& Craft, 1970); that the shortening of visual RT decreases as the interval between the imperative and accessory stimulus increases (Bernstein, Clark, \& Edelstein, 1969b; Bernstein et al., 1969a; Michie, Clark, Sinden, \& Glue, 1976; Morrell, 1967, 1968a, 1968b; Posner, Nissen, \& Klein, 1976; Wadman, Boerhout, \& Denier

Supported in part by Grant BNS 80-23125 from the National Science Foundation to the second author. S.C.A.M. Gielen and P.J.M. van den Heuvel are associated with the Department of Physiological and Medical Physics at Rijksuniversiteit Utrecht, and $R$. A. Schmidt is associated with the Department of Kinesiology at UCLA. van der Gon, 1980; Erkelens, Note 1); that the facilitation applies for various accessory-stimulus modalities, such as shock (Michie et al., 1976; Semjen, Bonnet, \& Requin, 1973; Todd, 1912) and torques applied to the responding member (Gurfinkel \& Pal'tsev, 1965; Wadman et al., 1980; Erkelens, Note 1), and for visual accessories in shortening auditory RT (Morrell, 1968a; Posner et al., 1976).

Various hypotheses have been advanced to account for these results. The earliest findings (e.g., Hershenson, 1962; Todd, 1912) were based on paradigms without catch trials. That is, on some proportion of the trials, an accessory stimulus would be presented along with the imperative stimulus. Although the subject was to respond only to the imperative stimulus, the subject was, in fact, free to respond to either of them, since there were no catch trials on which the accessory was presented alone (and on which the subject should withhold a response). The simple hypothesis that the RT is determined by the more rapid of the two modalities appeared to be contradicted by evidence that the combined RT (imperative plus accessory) was shorter than the RT for the accessory alone plus the delay between the imperative and accessory. But Nickerson (1973) and Raab (1962) have argued that there may be a kind of statistical facilitation present in these paradigms, due to the variability in "arrival times" of the results of processing in the two modalities at some later stage of processing that leads to a response. ${ }^{1}$ In Raab's model, no integration of sen- 
sory modalities is assumed; rather, the facilitation of RT is based on the subject's using that modality which, for a particular trial, happens to provide the earliest indication that some stimulus was presented.

Raab's model uses the following logic and mathematics. Since the subject responds to whichever of two stimulus modalities "arrives" first, the RT in combined-stimulus situations is based on the distribution of minima between the arrival times of the two stimulus modalities. This is shown in Figure 1, in which two stimulus modalities $S$ and $L$ have distributions of arrival times $S(t)$ and $L(t)$, shown on the left. The distributions of minima, $F(t)$, on the right, are calculated as:

$$
F(t)=S(t) \int_{t}^{\infty} L(t) d t+L(t) \int_{t}^{\infty} S(t) d t .
$$

When $S(t)$ and $L(t)$ do not overlap very much, as shown in the top sets of curves, the mean of the distribution of $F(t)$ is equal to that of $S(t)$, providing no statistical facilitation. But when the two stimulus distributions overlap more, beginning when the mean of $L(t)$ is about 1 SD greater than that of $S(t)$, the mean of $F(t)$ is shortened. When the two distributions overlap completely, the greatest shortening of the mean of $F(t)$ is found, as shown in the lower panel; this provides maximal statistical facilitation. Notice that, even if the two stimulus modalities are matched in subjective intensity, so that their arrival times are equal, the statistical facilitation hypothesis predicts a
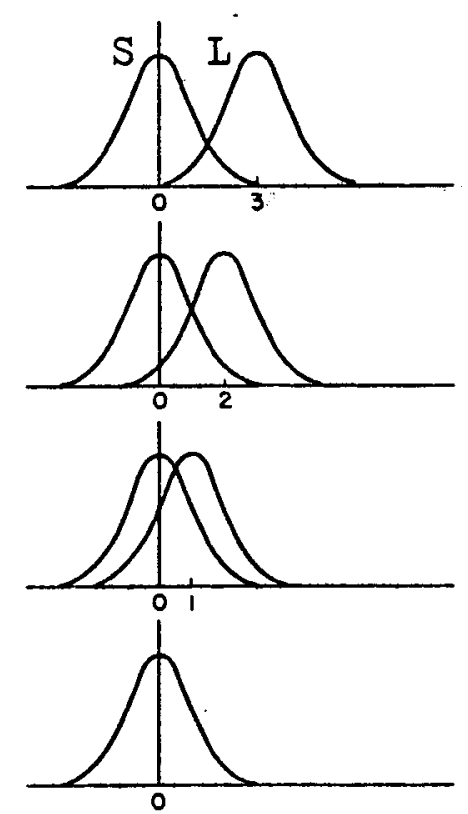

Figure 1. Statistical facilitation from Raab's model. [The distribution of minima, $F(t)$, shifts leftward as the overiap between the component distributions $S(t)$ and $L(t)$ increases; from Raab, 1962]. shortened RT for the combined events (e.g., Figure 1, lower curves).

Nickerson has argued that available data show more facilitation than can be accounted for by even this kind of statistical facilitation, leading to his conclusion that some "true" intersensory facilitation was taking place. These evaluations were admittedly difficult, though, as the variances of RTs for visual and accessory stimuli (necessary for use of the Raab model) were often not reported in the earlier literature, or were produced in designs that were, for a variety of reasons, inadequate (e.g., with "trimmed" means, etc.). And the estimated size of the intersensoryfacilitation effect (i.e., over and above the estimates of statistical facilitation) were usually quite small (e.g., $20 \mathrm{msec}$ ), leaving a strong possibility for errors in Nickerson's conclusions.

But Raab's method of estimating statistical facilitation, and, hence, Nickerson's conclusions based on it, has a number of difficulties, in our view. Raab assumed that the distributions of arrival times for the visual and auditory (accessory) channels were normally distributed, with equal variances. If these arrival times have similar distributions as the RTs for these channels, then there is good reason to think that the distributions of arrival times are positively skewed, with variance for vision being larger than that for audition and with variance for both vision and audition being larger than that for kinesthetic stimuli. These dissatisfactions with Raab's estimates of statistical facilitation lead to the major goal of this paper. With considerably relaxed assumptions about the nature of the distributions of arrival times, and about the relative sizes of their variances, we have generated a modification of the Raab model, otherwise using the same logic as Raab did. When this model is applied to the RT data produced in our vision-alone vs. vision plus either auditory accessory (Experiment 1) or kinesthetic accessory (Experiment 2) situations, we have a basis for estimating the size of any statistical facilitation effects in a way we believe to be more effective than that used by Nickerson (1973) and Raab (1962). If our data show that the combined (visual plus accessory) RTs are shorter than our revised estimate of combined RTs based on statistical facilitation, then evidence for some "true" intersensory facilitation would be provided.

A second major concern of this paper is the nature of the experimental design for evaluating so-called intersensory-facilitation effects. Nickerson (1973), Posner (1978), and others have argued that designs with catch trials-where the accessory stimulus is occasionally presented alone, and the subject must not respond if it is-are to be preferred because they supposedly eliminate the possibility of statistical facilitation. The reasoning is that the subject is directed to respond to the imperative stimulus (or to combined- 
stimulus events) only, and thus attention is directed to the imperative stimulus, not to the accessory. In such cases, Nickerson argues that the response to an imperative-plus-accessory stimulus is based on an imperative stimulus speeded in some way by the accessory; he further argues that statistical facilitation could not occur, since the accessory stimulus would be, under such instructions, incapable of triggering the response if its arrival time happened to be shorter than that for the imperative stimulus.

While we agree that designs with catch trials make it unlikely for statistical facilitation to occur, we are concerned about them for essentially two reasons. First, it is perhaps possible for the subject to engage in complex switching strategies which would allow a kind of statistical facilitation. One such strategy would be to attend to both the imperative and accessory channels. If the imperative and accessory stimulus are presented, then the subject could respond to the (faster) accessory by switching attention to its processing, ignoring the imperative stimulus. If only the imperative stimulus is presented, presumably the subject would respond only on the basis of it, and a response could be withheld if only the accessory appeared. There is no good evidence that subjects can, in fact, accomplish these kinds of strategies, but the possibility of such processes is made considerably more plausible by the recent findings on automatic processing which show very rapid and attention-free responding to well-practiced stimulusresponse pairs (e.g., Schneider \& Fiske, 1982; Schneider \& Shiffrin, 1977; Shiffrin \& Schneider, 1977). The point is that the utilization of designs with catch trials might not guarantee that statistical facilitation cannot occur.

A second concern about designs with catch trials concerns certain "side effects" produced in such designs. One difficulty is that the estimates of RT to vision alone, when such trials are embedded within a series of other kinds of trials (e.g., catch trials, vision-plus-accessory trials), are usually inflated somewhat (Nickerson, 1973), reflecting the old idea that RT is not so much a function of the particular stimulus that was presented, but of that stimulus in relation to other possible stimulus events. This is important, because the size of the intersensory-facilitation effect is calculated by subtracting the RT for imperativeplus-accessory trials from the RT for imperative-alone trials, and thus the size of such effects could be somewhat overestimated in designs with catch trials. This is made more serious by the fact that the difference between the combined vision-plus-accessory RTs and the visual RTs (corrected for statistical facilitation) can be very small-often 10 to $20 \mathrm{msec}$ in our experiments. Also, experienced subjects tell us that they use different strategies in paradigms with and without catch trials. For all these reasons, we are uncomfortable with accessory-alone catch trials.
In sum, the available evidence for intersensory facilitation of RT is relatively weak. First, studies using no catch trials appear to show such effects, but there are problems in evaluating the size of the statistical-facilitation effects (if any) based on Raab's model. And second, while studies using accessoryalone catch trials appear to prevent statistical facilitation, we have argued that statistical facilitation is still possible and that such designs produce other problems that interfere with the interpretation in terms of intersensory facilitation. These concerns motivated the present experiments. If combined imperativeplus-accessory RT can be shown to be shorter than the RT to the imperative stimulus alone, with corrections for statistical facilitation using a modification of Raab's procedures, then a stronger basis for "true" intersensory facilitation of RT would be provided.

\section{EXPERIMENT 1}

\section{Method}

This study examined the possible intersensory facilitation of visual RT, using auditory accessory stimuli. These experiments are similar to a number of studies reported in the literature, except that (1) statistical facilitation was estimated using our modifications of Raab's model, and (2) RTs were estimated through the use of early kinematic features of the movement rather than by a keypress.

Subjects. Eight subjects (seven males and one female) associated with the Department of Medical Physics in Utrecht were used in this experiment. All had had some experience with the apparatus used, and with RT studies in general. They were not paid for their services and were not aware of the hypotheses under test.

Apparatus. A 110-cm-high metal frame, $2.2 \mathrm{~m}$ long, supported two v-pulleys at either end, their axles mounted in ball bearings; a continuous cable was strung tightly between them. The cable supported a bracket to which a padded metal wrist cuff could be attached; a firm attachment to the subject's wrist allowed horizontal movement. To one of the $v$-pulleys was attached a potentiometer to record position, as well as torque motors that could be activated to provide various kinds of resistance to movement. The apparatus is described more completely in Wadman et al. (1980).

Signals from the apparatus were fed to amplifiers, and resulting position and velocity signals were delivered to a 7-channel FM tape recorder and to an oscilloscope visible only to the experimenter. The position of the handle was also represented by a small mark on a large-sized oscilloscope screen placed $30 \mathrm{~cm}$ from the center of the metal frame and visible to the seated subject on the other side of the frame. A $10-\mathrm{mm}$-square patch could be presented on the screen, and the patch could be made to "jump" $12.5 \mathrm{~cm}$ to the left under the experimenter's control. By moving the handle, the small mark would move continuously across the screen, with a 1:1 ratio of hand movement to mark movement. Subjects were instructed to move as quickly as possible, resulting in a movement time of approximately $80-100$ msec; thus, the subjects probably did not track the movement of the display, but rather preprogrammed the entire movement (e.g., Schmidt, 1982).

The oscilloscope display was $26 \times 36 \mathrm{~cm}$; it had a P4 phosphor, with an intensity decay time to $10 \%$ of about $1 \mathrm{msec}$. The rise time to $90 \%$ of maximum intensity was about $.5 \mathrm{msec}$. Displacement of the target patch on the screen occurred within $20 \mu \mathrm{sec}$. The target patch had a luminance of about $600 \mathrm{~cd} / \mathrm{m}^{2}$, and the background luminance of the oscilloscope screen was $10 \mathrm{~cd} / \mathrm{m}^{2}$. A normal loudspeaker was used to generate the auditory stimulus. 
It had a flat frequency response from 100 to $2000 \mathrm{~Hz}$ and a rise time shorter than $1 \mathrm{msec}$.

Procedures. A simple-RT task was used with a movement distance of $12.5 \mathrm{~cm}$ leftward to the new target position. A ready signal (Klaar in Dutch) was provided, followed in 1, 2, or $3 \mathrm{sec}$ (at random) by the movement of the patch on the oscilloscope. The subjects were to move to the target position as quickly as possible after the onset of the light; there was no emphasis placed on target accuracy.

There were three conditions, grouped into blocks that were presented in a different random order for each subject. First, there was a vision-alone condition, in which only the visual information was provided. Second, there was an auditory condition, in which a loud "pop" from a speaker adjacent to the subject's head provided the imperative stimulus; in this case, the visual information was two patches on the screen, one for the starting position and one for the target position, and there was no change in the visual array with the presentation of the auditory stimulus. Third, a combined condition presented the auditory "pop" delayed $65 \mathrm{msec}$ from the visual stimulus; the subjects were instructed to respond to the visual stimulus, the sound stimulus, or to both. All subjects received blocks of 25 trials of each condition, and the entire treatment order was experienced twice by each subject, providing a total of $\mathbf{5 0}$ trials for each subject for each condition. No catch trials of any kind were provided.

Modifications to Raab's model. Of primary concern in the present experiment was the comparison between the vision-alone RT and the vision-plus-auditory $R T$, with a shorter $R T$ in the second condition perhaps indicating facilitation. But, in these designs without catch trials, some estimate of statistical facilitation of combined-stimulus events is required to rule out the hypothesis that the subjects were simply responding to the more rapid of the two stimulus modalities. We, therefore, used Raab's model, but modified it in the following ways. Rather than assuming, as Raab did, that the distributions of the visual and auditory arrival times at some late processing stage had normal distributions with equal variances, we assumed that the shapes of the distributions of arrival times would be more effectively represented by the shapes of the distributions of the RTs to those stimulus events when presented alone. Thus, based on each subject's data in the visionalone and audition-alone conditions, estimates of the distributions of arrival times for each subject and modality were provided. The distributions of the arrival times of the visual and auditory modalities, analogous to $S(t)$ and $L(t)$ in Figure 1, were represented in the computer as histograms. Then, using the procedures described by Raab (and in Equation 1), we calculated the distribution of minima, $F(t)$. The only difference in computation was that the areas under the curves [e.g., $\int_{t}^{\infty} S(t)$, etc.] were computed digitally here, rather than continuously as in the example provided earlier. The mean of the distribution of minima, or $\overline{\mathrm{F}(t)}$, was taken as the estimate of the predicted combined RT when only statistical facilitation was operating. Then, to provide an estimate of the "true" intersensory facilitation effect, the theoretical combined RTs based on the distribution of $F(t)$ were compared with the obtained RTs from the combined visual and auditory conditions.

Measurement of reaction times. The tape-recorded trials were played onto an oscilloscope for scoring. RTs were taken from the record of the stimulus movement to the point at which velocity became nonzero. This resulted in measured RTs that were shorter (40 msec in our data) than they would have been had the time to first movement been measured and probably somewhat shorter than they would be in studies that use a switch closure to signal the end of RT.

\section{Results}

The average RTs for each of the subjects ${ }^{2}$ in the various conditions are shown in Table 1 . With all subjects averaged together, the RT for vision alone $(220.2 \mathrm{msec})$ was about $24 \mathrm{msec}$ longer than that for audition alone ( $186.6 \mathrm{msec})$, and the RT for the combined visual and auditory condition was intermediate (204.1 msec). This ordering was present for each of the eight subjects, and the effect considering all eight subjects in a repeated measures ANOVA was significant, with $F(2,10)=71.6, p<.05$.

A major concern in this experiment was the contrast between the combined visual and auditory RTs and the predicted combined RTs, the latter being based on our modifications of the Raab (1962) model. The predicted combined RTs are also given in Table 1, with each subject's value being based on all 50 visual trials and all $\mathbf{5 0}$ auditory trials. We also computed separate estimates based on the 25 oddnumbered trials and on the 25 even-numbered trials, in each modality. The average difference between these two estimates gives an indication of the stability of our 50-trial estimates. The average difference between the two estimates was $5.8 \mathrm{msec}$, and the differences between separate estimates ranged from .2 to $10.4 \mathrm{msec}$ for various subjects. The small average differences between the two estimates indicate that the model produced reasonably stable estimates of the predicted combined RTs.

Table 1

Mean Reaction Times and Within-Subject SDs for Various Subjects and Conditions in Experiment 1

\begin{tabular}{lllllllr}
\hline & \multicolumn{4}{c}{ Obtained RTs } & \multicolumn{2}{c}{ Predicted RTs } \\
\cline { 2 - 7 } Subject & RT-V & SD & RT-A & SD & RT-VA & SD & RT-VA \\
\hline 1 & 214.6 & 27.5 & 145.7 & 11.5 & 183.3 & 18.5 & 206.9 \\
2 & 204.8 & 22.2 & 150.8 & 16.2 & 183.6 & 12.3 & 197.7 \\
3 & 210.6 & 16.4 & 171.4 & 18.2 & 183.6 & 13.6 & 208.0 \\
4 & 218.9 & 14.9 & 185.4 & 19.5 & 210.4 & 13.5 & 216.5 \\
5 & 225.8 & 26.7 & 206.9 & 30.1 & 215.6 & 25.1 & 22.3 \\
6 & 199.3 & 17.6 & 168.7 & 18.9 & 187.0 & 13.8 & 198.1 \\
7 & 239.3 & 21.8 & 231.0 & 29.8 & 232.6 & 16.5 & 238.1 \\
8 & 248.2 & 31.2 & 233.2 & 22.9 & 237.9 & 18.5 & 245.5 \\
Mean & 220.2 & & 186.6 & & 204.1 & 216.6 \\
\hline
\end{tabular}

Note-RT-V = visual $R T ; R T-A=$ auditory $R T ; R T-V A=R T$ to combined visual and auditory stimuli. Predicted $R T-V A$ is the value predicted by the modified Raab model. 
For every subject, the obtained visual-auditory RT was shorter than that predicted by Raab's model, with these differences ranging from 5.5 to $23.6 \mathrm{msec}$. The average obtained visual auditory RT was $204.1 \mathrm{msec}$, and that predicted by Raab's model was $216.6 \mathrm{msec}$. In a repeated-measures ANOVA, this difference was statistically significant, with $F(1,7)=$ $21.7, \mathrm{p}<.05$. Thus, it seems clear that subjects were reacting to the combined visual plus auditory events more quickly than can be explained by a statisticalfacilitation model.

\section{Discussion}

The present data not only provide a replication of the earlier work on RTs to accessory stimuli, but they also suggest that the mechanisms involved in these reactions are not exclusively of the type termed statistical facilitation by Nickerson (1973) and Raab (1962). However, before turning to the theoretical implications of these data, it is of interest to determine whether the same kinds of conclusions can be made with other accessory stimuli. In particular, a number of previous experiments have used kinesthetic stimuli as accessories, with the general finding that the RTs to combined visual-plus-torque stimuli were more rapid than for vision-alone situations. Some of these studies have been directed at the concepts of intersensory facilitation (e.g., Michie et al., 1976; Semjen et al., 1973; Todd, 1912; Erkelens, Note 1), while others have used various kinesthetic stimuli in addition to visual stimuli to study spinal or attentional processes during RT to visual events (e.g., Gurfinkel \& Pal'tsev, 1965; Wadman et al., 1980). In either case, the results have been similar: when a kinesthetic stimulus (e.g., a torque to the responding limb, a shock, etc.) is provided during the early parts of visual RT, the response to the visual event is speeded relative to a no-accessory control condition.

Our concerns for Experiment 2 were to examine these combined visual and kinesthetic RTs in ways analogous to our treatment in Experiment 1, to determine the extent to which a statistical facilitation model (modified from Raab's model) could explain these kinds of findings. Although this question was motivated in part because of a desire to make the results from studies of statistical facilitation more generalizable, another reason for its inclusion here was that kinesthetic stimuli usually have much faster (perhaps 80 msec faster) RTs than do auditory stimuli (Chernikoff \& Taylor, 1952). This difference seems to be too large to be accounted for by the hypothesis that the kinesthetic stimuli were subjectively more intense than the auditory stimuli, and occurs even when the kinesthetic stimuli appear quite weak. Even so, allowing for the possibility that the kinesthetic stimuli may be inherently more rapid than auditory stimuli, it may be that these two stimulus modalities, when operating as accessories, may act in fundamentally different ways because of the differences in arrival times. For these reasons, Experiment 2 was patterned after Experiment 1, but with torque stimuli to the responding limb being used as accessories.

\section{EXPERIMENT 2}

This study was designed to examine the effects on visual RT of presenting a sudden kinesthetic stimulus (a torque to the responding limb) slightly after the visual imperative stimulus. As such, this study represents an extension of Experiment 1, using kinesthetic stimuli.

\section{Method}

Subjects. Two females and four males associated with the Department of Physiological and Medical Physics, University of Utrecht, were used as subjects. Two of the subjects had served in Experiment 1. All were well practiced at the task, but were not aware of the hypotheses under test.

Apparatus and Task. The apparatus and response were as described for Experiment 1, except for the following differences. The electric torque motors (Type PH" U12C) that "free-wheeled" in Experiment 1 were used here, activated by power amplifiers triggered by a delay mechanism initiated by stimulus onset. With this arrangement, a 20 -msec torque impulse of $20 \mathrm{~N}$, with a rise time of $3 \mathrm{msec}$, was applied to the wrist, acting in a direction opposite to the upcoming movement. A servomechanism was used for control, allowing well-defined force impulses to be applied. The onset of the pulse occurred $50 \mathrm{msec}$ after the onset of the imperative visual stimulus. The torque was of sufficient size to cause a slight movement of the handle (usually less than $1 \mathrm{~cm}$ ), and the subjective sensation was that a sharp tap had been delivered to the forearm. Visual stimuli were presented as before.

Procedures. The subjects were assigned to different randomized orders of three treatment conditions: vision alone, torque alone, and vision plus torque delayed by $\mathbf{5 0} \mathrm{msec}$. Each subject was run in two sessions on separate days, with each of the three treatment conditions being provided each day. Thus, the experiment involved 50 trials at each of the three treatment conditions, presented in a blocked fashion.

\section{Results}

The mean RTs for each condition and subject ${ }^{2}$ are shown in Table 2 . Considering values averaged across subjects, the RT to vision $(204.9 \mathrm{msec})$ was about $75 \mathrm{msec}$ longer than the RT to the torque stimulus $(129.3 \mathrm{msec})$, with the RT for the combined visual-plus-torque stimulus being intermediate $(164.7 \mathrm{msec})$. This general pattern of results was present for each of the six subjects, and the differences between means was significant in a repeated measures ANOVA, with $F(2,14)=19.9, p<$ .05 . These findings generally extended those for auditory accessories in Experiment 1.

A primary concern here was the extent to which these findings for combined torque and visual stimuli could be explained by a statistical facilitation hypothesis. Therefore, the distributions of torque-alone trials and vision-alone trials, for each subject separately, were used to determine a predicted combined RT based on our modification of Raab's model. 
Table 2

Mean Reaction Times and Within-Subject SDs for Various Subjects and Conditions in Experiment 2

\begin{tabular}{|c|c|c|c|c|c|c|c|}
\hline \multirow[b]{2}{*}{ Subject } & \multicolumn{6}{|c|}{ Obtained RTs } & \multirow{2}{*}{$\frac{\text { Predicted RTs }}{\text { RT-VK }}$} \\
\hline & RT-V & SD & RT-K & SD & RT-VK & $S D$ & \\
\hline 1 & 244.7 & 25.2 & 145.0 & 9.1 & 173.4 & 11.1 & 194.6 \\
\hline 2 & 209.7 & 30.2 & 130.8 & 8.9 & 164.1 & 10.0 & 179.2 \\
\hline 3 & 202.5 & 30.7 & 124.1 & 13.0 & 165.5 & 13.2 & 171.2 \\
\hline 4 & 165.5 & 13.1 & 116.5 & 11.9 & 142.8 & 7.7 & 156.0 \\
\hline 5 & 190.8 & 26.0 & 132.0 & 13.0 & 163.9 & 7.0 & 172.5 \\
\hline 6 & 216.6 & 23.7 & 129.6 & 9.8 & 178.2 & 11.1 & 193.2 \\
\hline Mean & 204.9 & & 129.3 & & 164.7 & & 177.8 \\
\hline
\end{tabular}

Note-RT.V = visual $R T ; R T-K=$ kinesthetic (torque) $R T ; R T-V K=R T$ to combined visual and kinesthetic stimuli. Predicted RT-VK is the RT predicted by the modified Raab model.

These predicted values are shown in Table 2, each of which is based on $\mathbf{5 0}$ vision and 50 kinesthetic trials. Separate determinations using odd- vs. even-numbered trials were done, as in Experiment 1. The average difference between the odd vs. even values found in these two determinations was $6.9 \mathrm{msec}$, with the differences for the various subjects ranging from 1.5 to $13.8 \mathrm{msec}$. Thus, while the average difference between parallel estimates here was somewhat larger than that found in Experiment $1(5.8 \mathrm{msec})$, the two estimates still show reasonable agreement and stability.

The major concern was a comparison of the obtained combined visual-plus-torque RTs vs. those predicted from the model. An examination of Table 2 shows that, on the average, the obtained RTs were $13 \mathrm{msec}$ shorter than predicted values. Further, similar differences were present for each of the six subjects, with the actual differences ranging from 5.7 to $21.2 \mathrm{msec}$. The results of a repeated measures ANOVA indicated that the 13-msec average difference was significant, with $F(1,5)=34.9, p<.05$. As before in Experiment 1, our data show that the combined visual-plus-torque RTs were faster than could be predicted from a statistical-facilitation model, and thus some other type of facilitation or intersensory integration, is required to explain the findings.

\section{DISCUSSION}

Our results seem relatively clear in suggesting that, under these dual-stimulus conditions, some kind of intersensory facilitation of RT is occurring. First of all, the statistical-facilitation hypothesis as proposed by Raab (1962), and as modified for the present purposes, cannot explain all of the reduced RT in the combined-stimulus conditions. Even when the rather restrictive and questionable assumptions of the Raab model-namely equal variances and normal distributions of the arrival times-were relaxed in the present case, a systematic analysis of the predicted vs. the obtained visual-accessory RTs showed that, for every subject, the responses to the combined-stimulus events were more rapid than could be predicted by the statistical-facilitation model. And we have shown these effects in designs without accessory-only catch trials in which (1) statistical facilitation seems most likely to occur and (2) difficulties associated with subject strategies and other "side effects" seem to be minimized. By default, then, these data suggest that some other kind of facilitation is occurring in these visual-accessory RTs, and a likely possibility is some kind of "true" intersensory interaction that leads to the speeded RTs.

In an excellent review, Nickerson (1973) has suggested two possible models to explain these kinds of effects of accessory stimuli. The historically oldest explanation is the energy-summation hypothesis, which holds that the energy from the accessory combines in some way with the energy for the imperative stimulus, leading to more rapid processing in early stages and to earlier arrival times. However, Nickerson presents a second preparation-enhancement hypothesis which argues that the accessory stimulus readies the later stages of processing in some way for the upcoming response. Nickerson's arguments, as well as those of Posner (1978), tend to favor the preparation hypothesis, but it is beyond the scope of the present discussion to go into these details (see Schmidt et al., Note 2).

What is the status of the statistical-facilitation hypothesis of Raab? A strong view of our data would be that, since the RTs were faster than could be accounted for by statistical facilitation, no statistical facilitation occurs at all, with all of the remaining enhancement of RT being due to either energysummation or preparation effects. This viewpoint would argue that the effect of the accessory stimulus in the present experiments should be estimated by the difference between the RT to the imperative stimulus and the RT to the combined event. In Experiment 1, for auditory accessories, this difference was $16 \mathrm{msec}$, while in Experiment 2, with kinesthetic accessories, this difference was about $\mathbf{4 0} \mathrm{msec}$. By varying the interval between the imperative stimulus and the onset 
of the accessory, these differences have become as large as $90 \mathrm{msec}$ in our unpublished data (Gielen, Schmidt, \& van den Heuvel, Note 3 ). This view is thus left with accounting for an intersensory effect of $90 \mathrm{msec}$ or more in certain instances, and such large effects of statistical facilitation seem unlikely. Furthermore, remember that the statistical-facilitation hypothesis holds that the subject responds to the stimulus with the earliest arrival time. ${ }^{1}$ In the case of kinesthetic accessories delivered simultaneously with a visual imperative stimulus, the subject would be expected invariably to react based on the (faster) kinesthetic modality. The 90 -msec "intersensory effect" mentioned above is probably best understood in this way: the subject simply responded to the kinesthetic channel, making the "visual RT" appear to be speeded by $90 \mathrm{msec}$ when the kinesthetic accessory was provided.

However, a much weaker view of these results is that statistical facilitation is always present in the way in which Raab has discussed-in that the subject responds to the stimulus with the earliest arrival time-but that there is some intersensory effect that operates over and above statistical facilitation to speed the RTs to the combined-stimulus events. In this case, the estimated size of the accessory stimulus is calculated as the difference between the RT for the combined stimulus events and the RT predicted by the modifications to Raab's model; these estimates are much smaller than with the stronger view just discussed. For the auditory accessories delayed by $65 \mathrm{msec}$, this effect size was estimated as $12 \mathrm{msec}$; for the kinesthetic accessories delayed by $50 \mathrm{msec}$, the effect was $13 \mathrm{msec}$.

We prefer the weaker of the above hypotheses, that statistical facilitation is always present to some extent, but the size of the effect will depend on the particular conditions used. For example, if the imperative stimulus is visual, and the accessory is kinesthetic (as in Experiment 2), then, if the accessory were presented simultaneously with the imperative stimulus, the subject would be expected to respond to the kinesthetic stimulus; in this case, the combined stimulus RT would be effectively equal to the RT for the kinesthetic-alone conditions, which is what we found in some earlier, unpublished, experiments (Gielen et al., Note 3). However, as the interstimulus interval is increased, with the kinesthetic following the visual stimulus by approximately the same amount as the difference between the RTs to these modalities alone (e.g., approximately $60-70 \mathrm{msec}$ ), then the distributions of arrival times would be assumed to overlap considerably. Here, then, the subject would be expected to respond to that stimulus modality which, on a particular trial, happened to arrive first. This kind of statistical facilitation would occur because the arrival times are variable from trial to trial, as Nickerson (1973) and Raab (1962) have suggested. But even with these two kinds of statistical facilitation, we argue that there must be some additional intersensory facilitation, in which the accessory speeds the processing of the imperative stimulus in some way. Or, more properly, since in this paradigm the labels "imperative" and "accessory" lose their meaning because the subject responds to whichever of them "arrives" first, we argue that the combined stimuli speed each other's processing in some way, leading to systematically earlier responding to the combined events than can be predicted by the statistical-facilitation hypothesis.

Certainly, more work is needed to be able to determine whether statistical facilitation and other intersensory effects are mutually exclusive processes, or whether under the conditions of the present experiment they can operate together to facilitate RTs. However, regardless of one's view of these issues about the statistical-facilitation hypothesis, our data argue that there can be some additional effect of combined-stimulus situations-not dependent on statistical facilitation-that speeds RT, and that some kind of intersensory facilitation (energy summation or preparation enhancement) is the basis of the speeded RT.

\section{REFERENCE NOTES}

1. Erkelens, C. Unpublished data, Faculty of Dentistry, Rijksuniversiteit Utrecht, 1981.

2. Schmidt, R. A., Gielen, S. C. A. M., \& van den Heuvel, P. J. M. On the locus of intersensory facilitation of reaction time. Manuscript submitted for publication May 1983.

3. Gielen, S. C. A. M., Schmidt, R. A., \& van den Heuvel, P. J. M. Unpublished experiments, Department of Physiological and Medical Physics, Rijksuniversiteit, Utrecht, 1981.

\section{REFERENCES}

Bernstein, I. H., Clakk, M. H., \& Edelstein, B. A. Effects of an auditory signal on visual reaction time. Journal of Experimental Psychology, 1969, 80, 567-569. (a)

Bernstein, I. H., Clark, M. H., \& Edelstein, B. A. Intermodal effects in choice reaction time. Journal of Experimental Psychology, 1969, 81, 405-407. (b)

Bernstein, I. H., \& Edelstein, B. A. Effects of some variations in auditory input upon visual choice reaction time. Journal of Experimental Psychology, 1971, 87, 241-247.

Chernikoff, R., \& TAYLOR, F. V. Reaction time to kinesthetic stimulation resulting from sudden arm displacement. Journal of Experimental Psychology, 1952, 43, 1-8.

Gunfinkel, V. S., \& PaL'Tsev, Y. I. Effect of the state of the spinal cord on the execution of a simple motor reaction. Biophysics, 1965, 10, 944-951.

Hershenson, $M$. Reaction time as a measure of intersensory facilitation. Journal of Experimental Psychology, 1962, 63, 289-293.

Michie, P. T., Clarke, A. M., Sinden, J. D., \& Glue, L. C. T. Reaction time and spinal excitability in a simple reaction time task. Physiology \& Behavior, 1976, 16, 311-315.

Morrell, L. Intersensory facilitation of reaction time. Psychonomic Science, 1967, 8, 77-78.

MorRell, L. K. Cross-modality effects upon choice reaction time. Psychonomic Science, 1968, 11, 129-130. (a) 
Morrell, L. K. Temporal characteristics of sensory integration in choice reaction times. Journal of Experimental Psychology, 1968, 77, 14-18. (b)

Nickerson, R. S. Intersensory facilitation of reaction time: Energy summation or preparation enhancement? Psychological Review, 1973, 80, 489-509.

Posner, M. I. Chronometric explorations of mind. Hillsdale, N.J: Wiley, 1978.

Posnen, M. I., Nissen, M. J., \& Klein, R. Visual dominance. An information-processing account of its origins and significance. Psychological Review, 1976, 83, 157-171.

RAAB, D. H. Statistical facilitation of simple reaction times. Transactions of the New York Academy of Sciences, 1962, 24, 574-590.

Schmid, R. A. Motor control and learning: A behavioral emphasis. Champaign, Ill: Human Kinetics Press, 1982.

Schneider, W., \& Fiske, A. D. Attention theory and mechanisms for skilled performance. In R. A. Magill (Ed.), Memory and control of motor behavior. Amsterdam: North-Holland, 1982.

Schneider, W., \& Shiffrin, R. Controlled and automatic human information processing: I. Detection, search, and attention. Psychological Review, 1977, 84, 1-66.

Semjen, A., Bonnet, M., \& Requin, J. Relationship between the time-course of Hoffman reflexes and the foreperiod duration in a reaction-time task. Physiology \& Behavior, 1973, 10, 1041-1050.
Shiffrin, R. M., \& Schneider, W. Controlled and automatic human information processing: II. Perceptual learning, automatic attending, and a general theory. Psychological Review, $1977,84,127-190$.

Simon, J. R., \& Craft, J. L. Effects of an irrelevant auditory stimulus on visual choice reaction time. Journal of Experimental Psychology, 1970, 86, 272-274.

Todn, J. W. Reaction time to multiple stimuli. Archives of Psychology, 1912, 3, 1-65.

Wadman, W. J., Boerhout, W., \& Denien van der Gon, J. J. Responses of the arm movement control system to force impulses. Journal of Human Movement Studies, 1980, 6, 280-302.

\section{NOTES}

1. The term "arrival time" will be used here and elsewhere in this paper to mean the time at which some early stage of (sensory) information processing delivers its results to a later stage that organizes a response. This use of the term is consistent with that by Nickerson (1973) and Raab (1962).

2. All of the analyses reported here were also conducted with median RTs. In all cases, the size of the effects, and their statistical significance, were essentially the same as with the analysis of mean values.

(Manuscript received May 21, 1982;

revision accepted for publication March 24, 1983.) 